\title{
Determination of Structural Properties of Some Important Polymers Used as Interfacial Layer in Fabrication of Schottky Barrier Diodes (SBDs)
}

\section{Çiğdem BİLKAN ${ }^{1 *}$}

\begin{abstract}
In this study, structural, electronic and thermal properties of important polymers such as Perylene (PER), Polypyrrole (PPy) and Polyvinyl alcohol (PVA) commonly used in the production of metal-polymer-semiconductor (MPS) type Schottky Barrier Diodes (SBDs) were determined. Since the opto-electronic properties of the materials depend on the electronic band gap, the High Occupied Molecular Orbital (HOMO) and Low Unoccupied Molecular Orbital (LUMO) energies for the polymeric structures and the gap between of energy levels were calculated. In addition, entropy, heat capacity and total thermal energy values were calculated over a wide temperature range and it was determined how thermochemical properties of polymers were affected with temperature. The obtained results showed that while PER has a planar structure, PPy and PVA has a non-planar structure. PER also has the highest chemical reactivity among the polymers examined with large band gap calculated as 3.03 $\mathrm{eV}$. In addition, thermochemical parameters of all polymers increase with increasing temperature almost as linearly.
\end{abstract}

Keywords: Schottky Barrier Diodes, Density Functional Theory (DFT), polymers, structural properties, HOMO-LUMO

${ }^{1}$ Çiğdem BİLKAN (Orcid ID: 0000-0002-3347-673X), Çankırı Karatekin Üniversitesi, Fen Fakültesi, Fizik Bölümü, Çankırı, Türkiye

*Sorumlu Yazar/Corresponding Author: Çiğdem BİLKAN, e-mail: cigdembilkan@gmail.com

Geliş tarihi / Received: 04-09-2019

Kabul tarihi / Accepted: 04-12-2019 


\section{INTRODUCTION}

Schottky barrier diodes (SBDs) with and without an interfacial layer are electronic devices formed by contacting metal and a semiconductor with one another at the lowest possible resistance (zero resistance in ideal case) at high temperature and low pressure. They are quite important in many semiconductor-based electronic devices manufacturing industries such as solar cells (SCs), photodetectors (PDs) and field effect transistors (FETs) [Bilkan, 2016]. While the interfacial layer is formed in the production of SBD, structural defects and impurities may occur that alter the energy band structure of the semiconductor and affect the device performance [Bilkan, 2016]. For this reason, recently various organic (polymer) layers such as rhodamine-101, poly(aniline), tetraamide-I, phenolsulfonphthalein, chitin, $\beta$-carotene, safranin T, PVA, $\mathrm{P}_{2}$ CIAn, PEDOT, polyindole, PER, and PPy are used to isolate metal and semiconductor from MS contacts, regulate charge transitions between them and reduce leakage current. If any of these polymers are placed at the metal/semiconductor interface, the MS type SBD converts to the MPS type SBD.

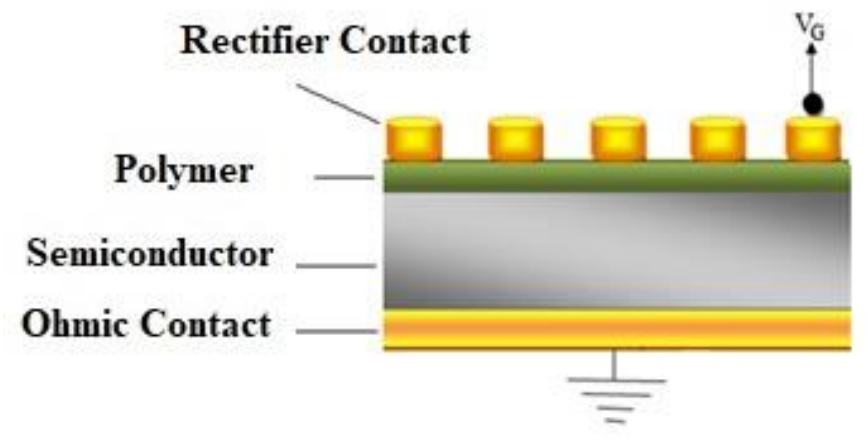

Figure 1. Schematic representation of SBD with polymer interfacial layer

Since the electrical properties of SBDs are often determined by their interface properties, determination of chemical and physical properties of the used polymers in SBDs is very important for scientific and industrial applications [Bilkan et al., 2016; Bilkan et al., 2017]. Unfortunately, in the literature, the number of studies in which the structural and electronic properties of polymers used in SBD fabrication are discussed in detail is quite limited. In order to fill this gap in the literature, the structural and electronic properties of the polymers have been investigated in detail in this paper.

Actually, since polymeric structures continue in the form of long chains, it is quite difficult to examine them theoretically. It is, therefore, more reasonable to consider a certain portion of the polymers for investigations. These type structures have been named as an oligomer [Jenkins et al., 1996]. For this study, octamer structures were taken into consideration in the investigation of PVA and PPy oligomers. Octamer modeling is a useful approach to understand the electronic and structural properties of polymeric systems and it has been used to investigate the structures of some polymers in the literature [Zgou et al., 2008]. To start with, the octamer structures of the oligomers have been optimized and the energies of the structures have been calculated. Later, HOMO and LUMO energies and thermochemical properties of the polymers have also been obtained from optimized octamer structures using the DFT method. The HOMO and LUMO are important parameters in determination of physical properties of chemical compounds. The energy gap between HOMO and LUMO energies is a considerable parameter in determining molecular electrical transport properties. The energy of the HOMO is directly related to the ionization potential, and LUMO energy is directly related to the electron affinity. This is also used by the frontier electron density for estimating the most reactive position in p-electron systems and also 
explains several types of reaction in conjugated system [Al-Saadi et al., 2015; Bilkan, 2019]. The HOMO-LUMO energies and the gap between HOMO and LUMO levels are also used to the description characteristic electronic properties of the semiconductor. Charge transport relates to the ability of charge carriers to move from one molecule to another; this depends on the energy gap between HOMO and LUMO. The conduction band and the valance band are usually replaced by LUMO and HOMO, respectively. [Y1ldirım, 2017]. The results obtained were discussed and many structural and electronic properties of the polymers were elucidated in detail.

\section{MATERIAL AND METHOD}

Three-dimensional geometries of the investigated polymers were drawn using Gaussview visualization program [Dennington et al., 2008]. Initially, the polymeric structures were optimized with a calculation level of DFT/6-311G(d,p). Using the optimized structures, HOMO-LUMO energies were calculated and the contour maps were drawn. The time-dependent DFT method (TD-DFT) was used for calculation of the electronic absorption spectra. Entropy and heat capacities of the structures were calculated with the same calculation level in a wide temperature range. All calculations were performed on a high-capacity computer by using Gaussian program [Frisch et al., 2004].

\section{RESULT AND DISCUSSIONS}

\section{Structural properties}

In order to determine the structural parameters and the electronic properties, at first, it is necessary to determine the optimized structures. In this study, three-dimensional structures of PER, PVA, and PPy were optimized by using DFT/B3LYP method and resulting structures were given in Figure 2.

a)

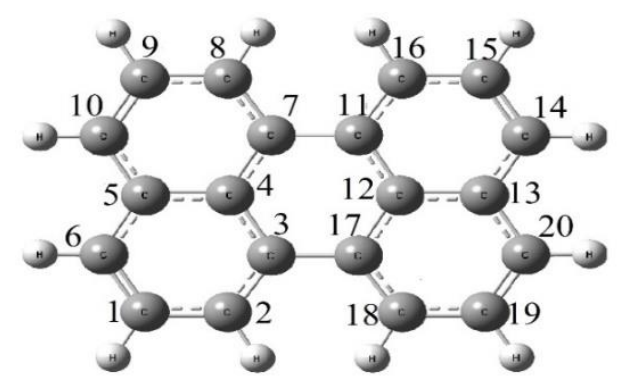

b)

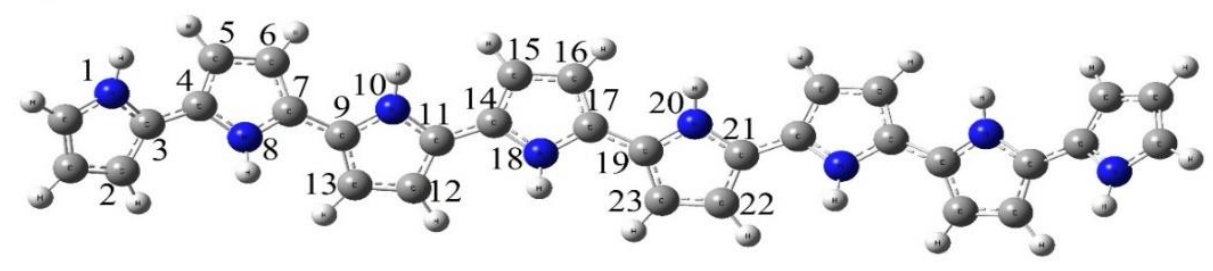

c)

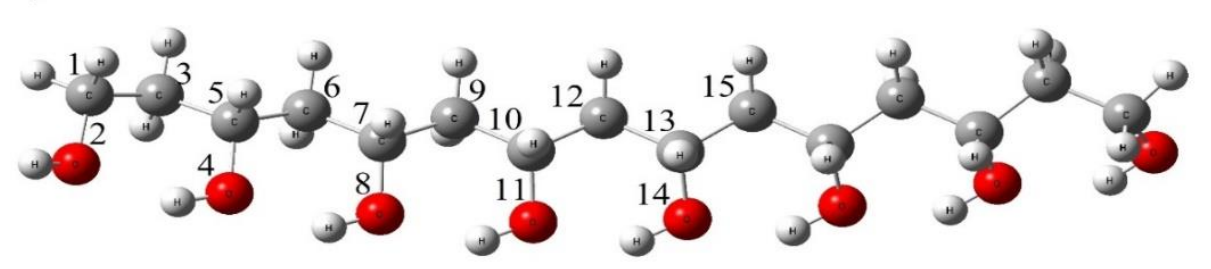

Figure 2. The optimized polymeric structures of a) PER b) PPy and c) PVA

In this part of the study, dihedral angles are examined to determine the structure of polymers because dihedral angles represent the angle between the two planes and provide important information 

Schottky Barrier Diodes (SBDs)

in predicting the shape of any chemical structure. For PER, $1 \mathrm{C}-2 \mathrm{C}-3 \mathrm{C}-17 \mathrm{C}, 2 \mathrm{C}-3 \mathrm{C}-4 \mathrm{C}-7 \mathrm{C}, 18 \mathrm{C}-17 \mathrm{C}$ 3C-4C, 15C-16C-11C-7C and 9C-8C-7C-11C dihedral angles were calculated as 180.0, -180.0, 180.0, 180.0 and $-180.0^{\circ}$ respectively, which indicates that the structure is planar. The $\mathrm{C}-\mathrm{C}$ bonds for PER were calculated as $1.37 \AA, \mathrm{C}=\mathrm{C}$ bonds as $1.43 \AA$. $7 \mathrm{C}-11 \mathrm{C}$ and $3 \mathrm{C}-17 \mathrm{C}$ bond lengths were calculated as 1.48 $\AA$, all of which are consistent with the experimental data found in the literature [Donaldson et al., 1953]. For PPy, N-C bond lengths were calculated between $1.38 \AA$ and $\mathrm{C}-\mathrm{C}$ bond lengths were calculated between 1.38-1.45 $\AA$. For PVA, C-C bond lengths were found to be 1.53-1.54 $\AA$ and C-O bond lengths were $1.43 \AA$. These values are quite similar to the results of previous calculations [Bala et al., 2015]. Furthermore, according to these results, PPy and PVA oligomers have a non-planar structure. Atomic charges are determinative in the formation of geometric parameters of a chemical compound. Whether the compound is in planar or nonplanar geometry is directly related to charges. In addition, whether the geometric structures are planar or not can trigger intermolecular interactions, which directly affect the electronic properties of the diode by reducing the gap between the HOMO-LUMO energy levels of the polymers used as the interfacial layer. The effect of planar geometric structure on electronic properties will be discussed in the following title electronic properties.

\section{Electronic properties}

In this paper, HOMO and LUMO energies for PER, PPy and PVA and the gap between of them were also calculated. Calculated parameters are given in Table 1. The band gap were calculated by $E g=$ $E_{\text {LUMO- }}$ HOMO. $_{\text {HOM }}$

Table 1. Calculated HOMO, LUMO and LUMO-HOMO energies (eV) of the polymers

\begin{tabular}{lccccc}
\hline & HOMO & LUMO & HOMO-1 & LUMO+1 & Gap \\
\hline PER & -5.21 & -2.18 & -6.81 & -0.79 & 3.03 \\
PPY & -4.31 & -0.91 & -4.71 & -0.57 & 3.40 \\
PVA & -6.36 & 0.05 & -6.91 & 0.78 & 6.31 \\
\hline
\end{tabular}

Determining HOMO-LUMO energies and gap between the two is important because these parameters provide the ability of electron-hole transport and a reasonable qualitative indication of excitation properties [Kaya, 2018]. The calculated band gaps were obtained as $3.03 \mathrm{eV}$ for PER, 3.40 $\mathrm{eV}$ for PPy and $6.31 \mathrm{eV}$ for PVA, respectively. The experimental band gap for PPy is around $2.83 \mathrm{eV}$ [Chakrabarty, 2014]. The experimental band gap value of PVA is $6.18 \mathrm{eV}$ [Abdullah et al., 2015]. As a result of our investigations, there are no experimental bandgap value of PER in the literature, but there are some studies in which the bandgap value was calculated for PER. One of these is the study conducted by Mohamad et al. [Mohamad et al., 2017], and the band gap value for PER was calculated to be approximately $2 \mathrm{eV}$. Although this value is close to the calculated value in our study, the reason why it cannot be the same is they used different calculation levels in their study.

For a chemical compound, the small energy gap means mostly high chemical reactivity and low kinetic stability because of narrow energy gaps in electronic transitions. This type compounds also termed as soft molecules [Fleming, 1976]. The computed band gaps of the oligomers were calculated very close to the experimental values in the literature. However, since the periodic boundary conditions are neglected in theoretical calculations, it is expected that there will be some difference between the calculated and the experimental values. In some studies made, it was seen that the obtained results were closer to the experimental values with increasing oligomer length. [Kaya, 2018]. 
For PER, PPy and PVA, HOMO and LUMO contour maps are shown in Figure 3.

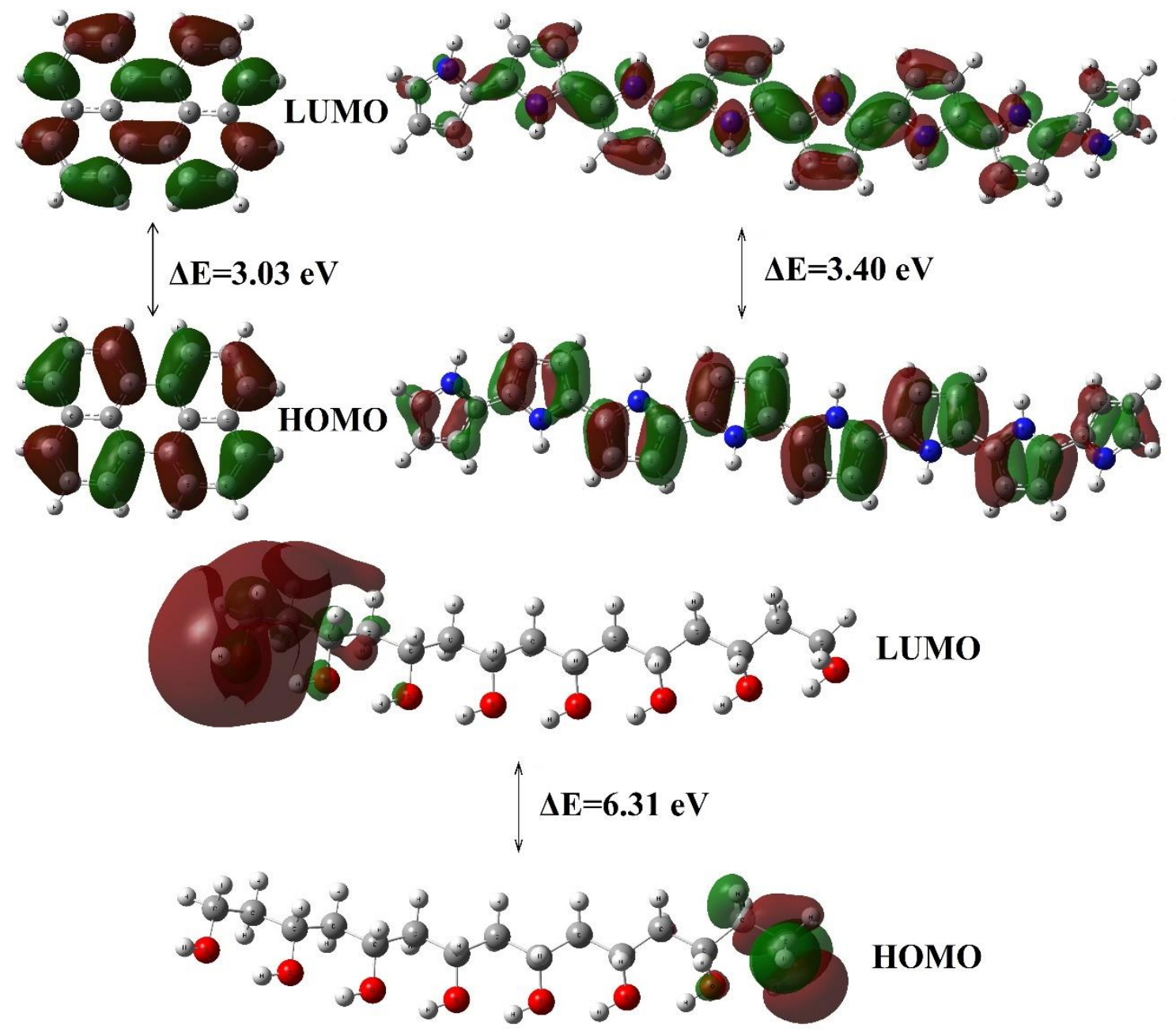

Figure 3. HOMO-LUMO contour maps and energy gap of PER, PPy and PVA

The absorption wavelengths, oscillator strength and excitation energies of the polymers were calculated and they were given in Table 2.

Table 2. The absorption wavelengths $\left(\lambda_{\max }\right)$, Oscillator strength and excitation energies of PER, PPy and PVA

\begin{tabular}{cccc}
\hline & PER & PPy & PVA \\
\hline$* \lambda_{\max }(\mathrm{nm})$ & $435.22(418.70)$ & $414.97(446.00)$ & $198.27(200.00)$ \\
Oscillator strength & 0.3509 & 2.2902 & 0.0000 \\
Excitation energy $(\mathrm{eV})$ & 2.8488 & 2.9878 & 6.2534 \\
\hline
\end{tabular}

*The experimental values of $\lambda_{\max }$ were given in brackets.

If molecular structures absorb light, the energy excites structure to a higher energy level. The wavelength of the light determines the type of excitation. If UV or visible light is emitted on the structure, electrons rise to the higher energy levels. The absorption spectrum of the compounds is the absorption of incoming light as a function of wavelength. The spectrum depends on its energy level structure and it is useful for identifying compounds. For the PER, absorption band was calculated at $435.22 \mathrm{~nm}$ with an oscillator strength of 0.3509 and calculated excitation energy is $2.8488 \mathrm{eV}$. In the literature, for PER, the experimental value of $\lambda_{\max }$ is $418.70 \mathrm{~nm}$ [Joblin et al., 1999]. Furthermore, the calculated $\lambda_{\max }$ of PPy 

Schottky Barrier Diodes (SBDs)

oligomer is $414.97 \mathrm{~nm}$. Chougule et al. observed to $\lambda_{\max }$ of PPy thin films at $446.00 \mathrm{~nm}$ in their experimental studies [Chougule et al., 2011]. Finally, $\lambda_{\max }$ of PVA were obtained at $198.77 \mathrm{~nm}$ in this study. The experimental value of $\lambda_{\max }$ was measured at $200.00 \mathrm{~nm}$ for PVA nanocomposite material [Nakhaei et al., 2012]. The results calculated in this study are very close to the experimental values in the literature.

\section{Thermochemical properties}

Determination of the thermochemical properties of chemical compounds and identification of behaviors of them under the temperature is quite important because these properties play an important role in the examination of the energy and heat associated with chemical reactions and physical transformations. Because of this, in Table 3, the entropy and heat capacities of the polymers in the wide temperature range of 100-400 were calculated together with the thermal energies at 1.0 atm pressure.

Table 3. Calculated thermochemical properties of the polymers in the wide temperature range of 100-400K.

\begin{tabular}{ccccc}
\hline & Temp. $(\mathrm{K})$ & Entropy & Heat Cap. & Thermal Energy \\
\hline 100 & 75.620 & 16.982 & 159.797 \\
150 & 84.834 & 25.363 & 160.848 \\
200 & 94.033 & 35.293 & 162.359 \\
PER & 250 & 103.501 & 46.086 & 164.392 \\
& 298.15 & 112.875 & 56.672 & 166.866 \\
& 300 & 113.239 & 57.076 & 166.971 \\
350 & 123.151 & 67.729 & 170.094 \\
PPy & 400 & 133.119 & 77.693 & 173.733 \\
\hline 100 & 132.176 & 50.696 & 330.971 \\
& 150 & 156.707 & 68.071 & 333.926 \\
& 200 & 179.599 & 88.497 & 337.830 \\
& 250 & 202.144 & 110.559 & 342.803 \\
& 298.15 & 223.810 & 132.023 & 348.644 \\
& 300 & 224.639 & 132.837 & 348.889 \\
& 350 & 247.049 & 154.253 & 356.072 \\
& 400 & 269.227 & 174.128 & 364.289 \\
\hline 100 & 109.588 & 42.232 & 307.520 \\
& 150 & 130.210 & 56.448 & 309.987 \\
& 200 & 148.966 & 70.740 & 313.165 \\
& 250 & 166.771 & 85.450 & 317.068 \\
& 300 & 183.416 & 100.082 & 321.533 \\
& 350 & 184.045 & 100.649 & 321.719 \\
& 201.020 & 115.865 & 327.133 \\
& 217.716 & 130.534 & 333.296 \\
\hline
\end{tabular}

*(Entropy and heat capacity is in Cal (Mol.K) ${ }^{-1}$, and thermal energy is in $\mathrm{Kcal} \mathrm{Mol}^{-1}$.

Heat capacity is one of the most important thermochemical parameters and is based on vibrational motions of molecular structures. Many other thermal parameters such as enthalpy, entropy and Gibbs free energy of a material can be calculated by utilizing heat capacity [Umesh Gaur and Bernhard Wunderlich, 1982]. Therefore, it is very important to determine the heat capacity for the polymers. As 
seen from Table 3, heat capacity and other thermochemical parameters of polymers with increasing temperature showed a linear increase. Thermochemical parameters have a direct effect on the vibrational between the atoms of chemical compounds. Alters in the vibrational modes of atoms can change the electronic properties of compounds shortly (with the effect of polarization). This may affect the compound's ability to perform intra and intermolecular interactions as previously mentioned and the electronic properties of the diode used as the interface layer. It is seen from results of a detailed investigation of the literature [Moraki et al. 2016; Gümüş and Altındal, 2014, Bilkan et al. 2017] for SBDs with PER, PPy, PVA interfacial layers, with increasing temperature the value of barrier height $\left(\Phi_{\mathrm{bo}}\right)$ increased whereas the value of ideality factor $(\mathrm{n})$ decreased. This is due to the effects of the changing temperature on both the semiconductor structure and the polymer layers.

\section{Applications of the polymers in SBDs}

The most important physical parameters for SBDs are rectification ratio (RR), saturation current $\left(I_{0}\right)$, $n$, series resistance $\left(R_{s}\right)$ and short circuit resistance $\left(R_{s h}\right)$. The conventional method for calculating these values is to interpret experimental current voltage (I-V) curves.
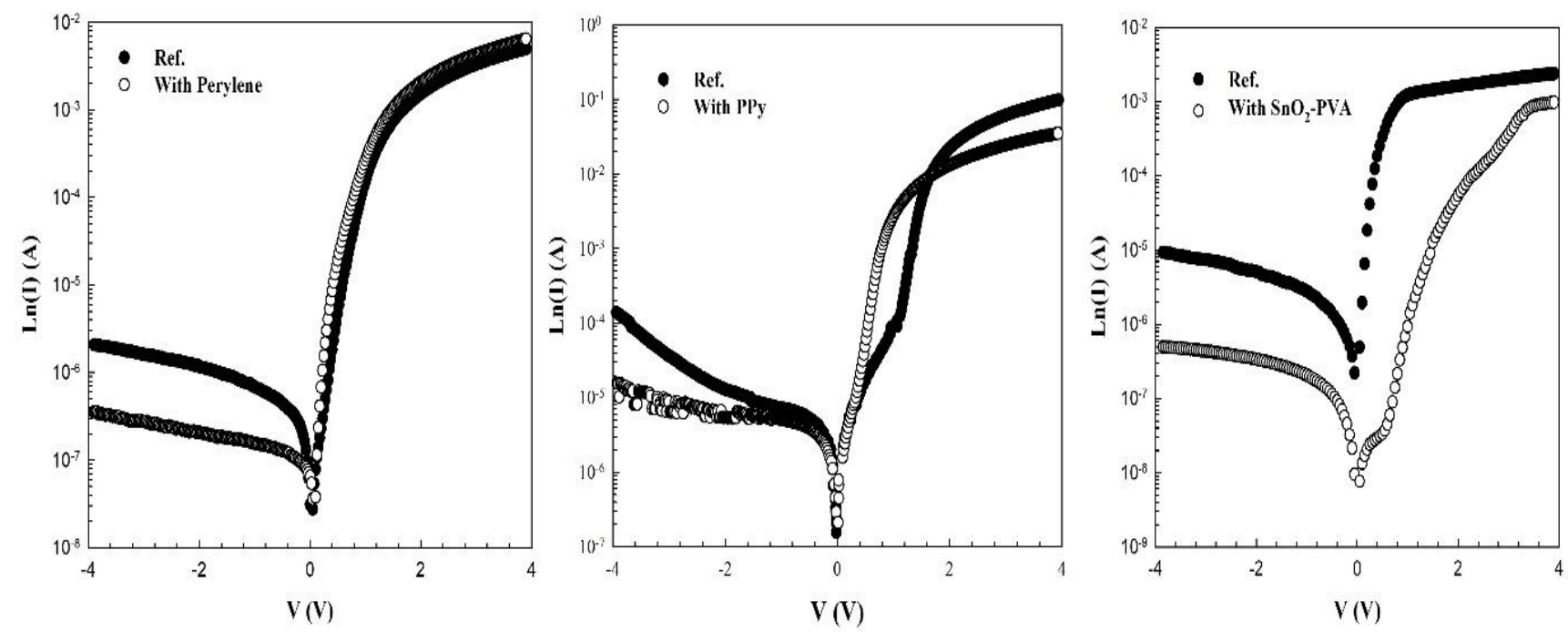

Figure 4. I-V characteristics of SBDs with and without PER, PPy and PVA interfacial layers.

Experimental I-V curves of SBD with PER, PPy, PVA interfacial layers were compared to ones without an interfacial layer, and they were given in Figure 4. The experimental data were taken from our previous studies [Bilkan, 2016; Bilkan et al. 2014 and Bilkan et al. 2019]. The characteristics of an ideal diode are: the diode must have a good rectification (RR high), be able to start early transmission ( $\mathrm{I}_{0}$ low), have an ideality factor close to 1 (ideally 1), low series resistance (ideally 0) and high short circuit resistance (ideally infinite). It is clear that the values of $n$ for diodes are considerably larger than unity. The high values of $n$ can be also attributed to the existence of interfacial layer, the particular density distribution of interface states $\left(N_{s s}\right)$ at $\mathrm{p}$-Si/PER interface and wide distribution of low SBH or patches at $\mathrm{Al} / \mathrm{p}-\mathrm{Si}$ interface. 
Determination of Structural Properties of Some Important Polymers Used as Interfacial Layer in Fabrication of Schottky Barrier Diodes (SBDs)

Table 4. Comparison of some main diode parameters.

\begin{tabular}{c|cccccc}
\hline & \multicolumn{2}{c}{ PER } & \multicolumn{2}{c}{ PPy } & \multicolumn{2}{c}{ PVA } \\
\cline { 2 - 7 } & Without & With & Without & With & Without & With \\
\hline $\mathrm{RR}$ & $2.06 \times 10^{3}$ & $15.7 \times 10^{3}$ & 345 & 1730.7 & 249.5 & 249.5 \\
$\mathrm{I}_{\mathrm{R}}(\mathrm{A})$ & $1.61 \times 10^{-6}$ & $2.75 \times 10^{-7}$ & $2.32 \times 10^{-6}$ & $5.92 \times 10^{-7}$ & $2.46 \times 10^{-7}$ & $2.95 \times 10^{-9}$ \\
$\mathrm{n}$ & 3.8 & 2.8 & 5.62 & 3.3 & 1.97 & 7.15 \\
$\mathrm{R}_{\mathrm{S}}(\Omega)$ & 544 & 716 & 50.5 & 124.5 & 1643 & 3995 \\
$\mathrm{R}_{\mathrm{sh}}(\mathrm{M} \Omega)$ & 10.7 & 1.83 & 0.08 & 0.3 & 0.41 & 7.99 \\
\hline
\end{tabular}

The values obtained from the graphs for diode parameters were compiled into Table 4 . It can be clearly seen from the values in the table that the performance in MPS type SBDs are better than the MS type SBDs.

\section{CONCLUSIONS}

In this study, we have investigated structural, electronic and thermal properties of some important polymers such as Perylene, Polypyrrole and Polyvinyl alcohol used in the SBDs production. The reliability of the calculations was confirmed by comparing the results of the study with the experimental data in the literature. Since the conduction band, valance band and band gap, which are very important parameters for semiconductors, are the equivalents in the molecular structures, HOMO and LUMO energies and the gap between them are determined for each polymer. In addition, entropies, heat capacities and thermal energies of the polymers were calculated and their changes depend on temperature were investigated. As a result of the study, it is clearly seen that PER has a planar structure, PPy and PVA has a non-planar structure. Moreover HOMO-LUMO gap was calculated as $3.03 \mathrm{eV}, 3.40 \mathrm{eV}, 6.40$ eV for PER and $(n=8)$ oligomer structures of PPy and PVA, respectively. According to this result, PER exhibit higher chemical reactivity than other polymers investigated in this study. Furthermore, it is seen the increased temperature caused a linear increase in the heat capacity of the polymers.

\section{ACKNOWLEDGMENT}

The author would like to thank Dr. Mustafa Tuğfan Bilkan for his help in the calculations and contributions to the study. The author would like to thank the reviewers for evaluating the article in a careful and constructive manner.

\section{REFERENCES}

Abdullah OG, Aziz SB, Omer KM, Salih YM, 2015. Reducing the optical band gap of polyvinyl alcohol (PVA) based nanocomposite, Journal of Materials Science: Materials in Electronics, 26: 5303-5309.

Al-Saadi SR, Muthu AA. 2015. Vibrational spectroscopic studies, normal co-ordinate analysis, first order hyperpolarizability, HOMO-LUMO of midodrine by using density functional methods. Spectrochim Acta Part A, 134: 127-142.

Balaa Z, Tripathia SK, KumarR, 2015. DFT study of CdS-PVA film, International Conference on Materials Science and Technology (ICMST 2012), IOP Conf. Series: Materials Science and Engineering 73: 1-4.

Bilkan Ç, 2016. Polimer Arayüzey Tabakalı ve Tabakasız Schottky Engel Diyotların Elektriksel Karakteristiklerinin Karşılaştırılması, Gazi Üniversitesi Fen Bilimleri Enstitüsü, Doktora Tezi (Basılmış).

Bilkan Ç, Azizian-Kalandaragh Y, Altındal Ş, Shokrani-Havigh R, 2016. Frequency and voltage dependence dielectric properties, ac electrical conductivity and electric modulus profiles in $\mathrm{Al} / \mathrm{Co}_{3} \mathrm{O}_{4}-\mathrm{PVA} / \mathrm{p}-\mathrm{Si}$ structures, Physica B: Condensed Matter, 500: 154-160. 

Schottky Barrier Diodes (SBDs)

Bilkan Ç, Azizian-Kalandaragh Y, Sevgili Ö, Altındal Ş, 2019. Investigation of the efficiencies of the (SnO2PVA) interlayer in $\mathrm{Au} / \mathrm{n}-\mathrm{Si}$ (MS) SDs on electrical characteristics at room temperature by comparison, Journal of Materials Science: Materials in Electronics, 10: 1-10.

Bilkan Ç, Badali Y, Fotouhi-Shablou S, Azizian-Kalandaragh Y, Altındal Ş, 2017. On the temperature dependent current transport mechanisms and barrier inhomogeneity in $\mathrm{Au} / \mathrm{SnO}_{2}-\mathrm{PVA} / \mathrm{n}-\mathrm{Si}$ Schottky barrier diodes, Applied Physics A, 123: 560-570.

Bilkan MT, 2017, 2019. Quantum chemical studies on solvent effects, ligand-water complexes and dimer structure of 2,2'-dipyridylamine, Physics and Chemistry of Liquids, 57:1, 100-116.

Chakrabarty BS, 2014. Evaluation of optical constants of wide band gap cadmium doped polypyrrole, International Journal of Research in Engineering \& Technology, 2: 37-44.

Chougule MA, Pawar SG, Godse PR, Mulik RN, Sen S, Patil VB, 2011. Synthesis and Characterization of Polypyrrole (PPy) Thin Films, Soft Nanoscience Letters, 1: 6-10.

Dennington RD, Keith TA, Millam JM, 2008. GaussView 5, Gaussian, Inc.

Donaldson DM, Robertson JM, White JG, 1953. Proceedings of the Royal Society of London. Series A, Mathematical and Physical Sciences, 220: 311-321.

Fleming I, 1976. Frontier Orbitals and Organic Chemical Reactions, Wiley, London.

Frisch MJ, Trucks GW, Schlegel HB, Scuseria GE, Robb MA, Cheeseman JR, Montgomery JA, Jr., Vreven T, Kudin KN, Burant JC, et all, 2004. Gaussian, Inc., Gaussian 03, Revision D.01, Wallingford CT.

Gaur U, Wunderlich B, 1982. Advanced Thermal Analysis System (ATHAS) Polymer Heat Capacity Data Bank, Computer Applications in Applied Polymer Science, 197: 355-366.

Gümüş A, Altındal Ş, 2014. Current-transport mechanisms in gold/polypyrrole/n-silicon Schottky barrier diodes in the temperature range of 110-360 K, Materials Science in Semiconductor Processing, 28: 66-71.

Jenkins AD, Kratochvil P, Stepto RFT, Suter UW, 1996. Glossary of Basic Terms in Polymer Science, 68: 22872311

Joblin C, Salama F, Allamandola L, 1999. Absorption and emission spectroscopy of perylene „C20H12... isolatedin Ne, Ar, and N2 matrices, Journal of Chemical Physics, 110: 7288-7297.

Kaya A, 2018. Theoretical studies of structural, optic and electronic properties of polypyrrole (PPy) oligomer, Turkish Computational and Theoretical Chemistry, 2: 49-56.

Mohamad M, Ahmed R, Shaari A, Goumri-Said S, 2017. Structure-dependent optoelectronic properties of perylene, di-indenoperylene (DIP) isolated molecule and DIP molecular crystal, Chemistry Central Journal, 11: $125-134$.

Moraki K, Bengi S, Zeyrek S, Bülbül M, Altındal S, 2016. Temperature dependence of characteristic parameters of the $\mathrm{Au} / \mathrm{C}_{20} \mathrm{H}_{12} / \mathrm{n}$-Si Schottky barrier diodes (SBDs) in the wide temperature range, J Mater Sci: Mater Electron, 28:3987-3996.

Nakhaei O, Shahtahmassebi N, Rezaeeroknabadi M, Bagheri MM, Mohagheghi MMB, 2012 Synthesis, characterization and study of optical properties of polyvinyl alcohol/ $\mathrm{CaF}_{2}$ nanocomposite films, Scientia Iranica, 19: 1979-1983.

Y1ldirım M, 2017. Determination of Contact Parameters of Au/n-Ge Schottky Barrier Diode with Rubrene Interlayer, Journal of Polytechnic, 20:165-173.

Zgou H, Bouzzine SM, Bouzakraoui S, Hamidi M, Bouachrine M, 2008. Theoretical study of structural and electronic properties of oligo(thiophene-phenylene)s in comparison with oligothiophenes and oligophenylenes, Chinese Chemical Letters, 19:123-126. 